Zulassungsausschuss ... ist verpflichtet, auf Verlangen von Hochschulen oder Hochschulkliniken die Ambulanzen, Institute und Abteilungen von Hochschulkliniken (Hochschulambulanzen) zur ambulanten ärztlichen Behandlung der Versicherten ... zu ermächtigen."

Wenn auch diese systematisch-historische Auslegung die Bedeutung der Hochschule an sich weiter stärkt und somit tendenziell eine extensive Normauslegung bestätigt, so wirft die Umstrukturierung der Norm vom sog. Normalfall einer Ermächtigung im Sinne eines Antragsverfahrens in den Ausnahmefall einer Ermächtigung kraft Gesetzes rechtliche und tatsächliche Fragen auf, die eine restriktive Normauslegung zwar nahelegen würde, welche aber letztlich nicht haltbar ist.

Das Gebot der Rechtsklarheit fordert klare Regelungen. Dies gilt auch für das Rechtsinstitut der „Ermächtigung“, das - unabhängig der gesetzlichen Ausgestaltungen - gemäß \95 Abs. 3 SGB V zur vertragsärztlichen Leistungserbringung nicht nur berechtigt, sondern auch verpflichtet.

Vorliegender Fall verdeutlicht exemplarisch, dass mit der Normänderung zur gesetzlichen Ermächtigung die Leistungserbringung der außerklinischen HSA im Gegensatz zur klaren Struktur einer Hochschulklinik zuweilen ,,im Dunkeln“ liegen kann. Über die durch das Bay. LSG beantwortete Frage, dass die Ermächtigung des Instituts für
Pathologie nach $\$ 117$ SGB V im Rahmen der Prüfung des Bedarfs zu berücksichtigen ist, wäre sicherzustellen, ob die Bedarfsdeckung in der tatsächlichen Praxis auch erfolgt. Vor dem Stichtag der Gesetzesänderung, also bis zum 22.7.2015 hatte das pathologische Institut selbst kein Interesse an einer Ermächtigung, da es diese dann ja gemäß \$117 Abs. 1 SGB a. F.) beim Zulassungsausschuss beantragt hätte. Mit dem Inkrafttreten des GKV-VSG war es zwar ab dem 23.7.2015 zur Leistungserbringung kraft Gesetzes verpflichtet, genau diese Verpflichtung wurde aber offenkundig nicht umgesetzt.

Für den konkreten Fall wäre sowohl eine Beiladung des Instituts der Pathologie als auch der Hochschule zum Verfahren erhellend gewesen. Beigeladen waren ausschließlich der Ärztliche Leiter des Instituts der Pathologie als Antragsteller auf persönliche Ermächtigung und die Krankenkassen. Unter dem Aspekt, dass eine jede Ermächtigung zur Leistungserbringung berechtigt, aber auch verpflichtet, zeigt der Sachverhalt, dass zur Sicherstellung der vertragsärztlichen Leistungserbringung eine Registrierung der Hochschulambulanzen nach \$117 SGB V zur Umsetzung der Norm dringend erforderlich ist. Das ergibt sich auch in Bezug auf die notwendigen Bedarfsermittlungen, welche außerhalb des Regelungskreises des $\$ 117$ SGB V bei Anträgen auf Ermächtigungen erforderlich werden.

\title{
Absolution von Richtlinienverstößen oder untauglicher Versuch der Kanonisierung von Nicht-Recht?
} Ulrich Schroth und Elisabeth Hofmann

Original erschienen in Medizinrecht 35. Jahrgang, Heft 12, S. 948-950, https://doi.org/10.1007/s00350-017-4787-6

Professor Dr. iur. Ulrich Schroth,

Elisabeth Hofmann,

Ludwig-Maximilians-Universität München,

Prof.-Huber-Platz 2, 80539 München, Deutschland
Der Originalbeitrag wurde leider ohne die Autorenkorrekturen publiziert. Auf der Seite 950, rechte Spalte, Zeilen 6 und 11, muss es statt „,zwölf Jahre(n)“ richtig „22 Jahre(n)“ heißen.

Wir bitten, den Fehler zu entschuldigen. Der Verlag 